\title{
Status of digital administrative services in the Republic of Bulgaria
}

\author{
Katia Kirilova $^{1 *}$ \\ ${ }^{1}$ University of National and World Economy - Sofia, Bulgaria
}

\begin{abstract}
Digital administrative services are an essential component of the e-government system. They provide to citizens and businesses opportunities to facilitate contacts with the administration. To be developed and used, they need to be designed very carefully in advance. For this purpose, in most cases, modeling and reengineering of the main work processes is done. In this context, the study aims to present some of the results of a survey conducted among a sample of municipalities in the Republic of Bulgaria. The applied methods are related to: preparation and conducting of an online survey; methods related to data collection and storage by the respondents; statistical methods for analysis of the obtained results. The main results represent the degree of development of digital administrative services in the Republic of Bulgaria. They were obtained from the survey. The conclusions are in the direction of clarifying the degree of development of digital administrative services.
\end{abstract}

\section{Introduction}

The bodies of the central and local administration are exposed to constant changes in the economic environment and the regulatory framework. This is a good prerequisite for continuous improvement of administrative services. In this way, it realizes to the maximum extent the mission of the administration to benefit citizens and businesses. In this regard, with the expansion of administrative services, the quality and number of provided administrative services is increasing. Some of them have been digitized by the administrations, while others are still provided in traditional form. In theory and practice, there is no consensus on what percentage of digitized services for a particular administration. It is believed that in the digital age, the services offered should also be fully digitalized. However, this involves human capital and significant resources for effective administrative reform. The extent to which the individual municipalities in the Republic of Bulgaria are successful in this process can be successfully established from empirical studies. The results are a good basis for comparisons, both for the situation in different periods of time and between individual municipalities or groups of municipalities. This issue is at the heart of this study. To solve these problems, an appropriate methodology for conducting research is proposed, as well as the empirical results are analyzed.

\footnotetext{
* Corresponding author: katia.kirilova@unwe.bg
} 


\section{Research methodology}

The survey was conducted in compliance with the appropriate sequence of actions and steps. This process consists of:

- the development of a specialized software environment for communication with the respondents;

- building a database for communication with respondents;

- automated sending to the respondents information about the conducted survey.

The specialized software environment is built with the means of Visual Studio. Fig. 1 shows the main screen of the software application.

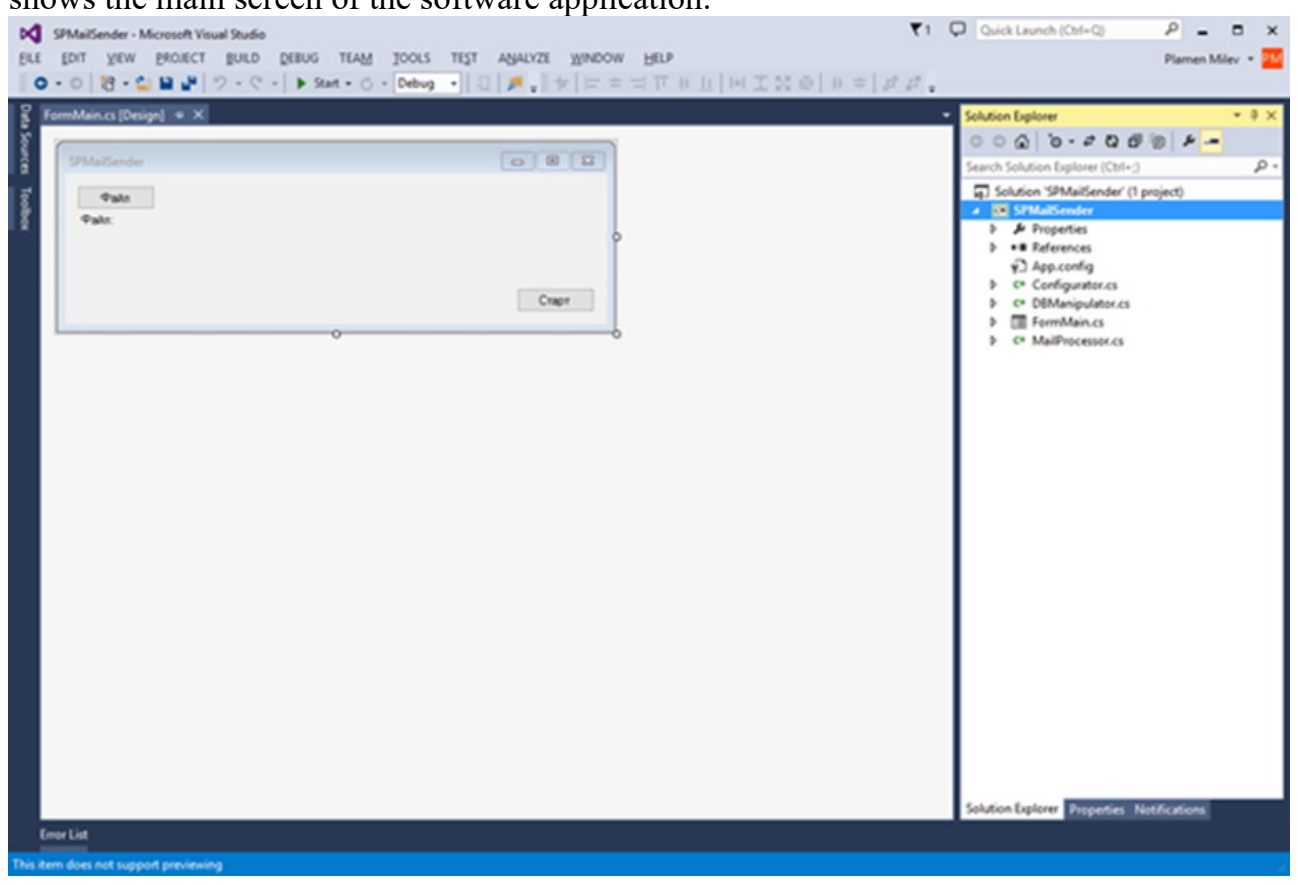

Fig. 1. Main screen of the software application

Building a database for communication with respondents is also an important task. The data with the contact information of the municipalities identified in the sample should be stored in it. In this way, conditions are created for the automated sending of information about the survey, reminiscent information, as well as results from conducted surveys. In Fig. 2 is given in graphical form the database with contacts, created in MySQL environment.

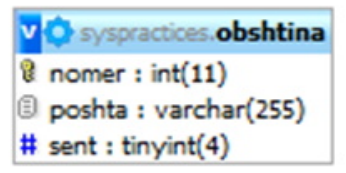

Fig. 2. Database with contacts of the respondents

After the construction of the software application and the respective database with contacts, conditions are created for the automated sending of information. Next, the respondents filled in the proposed questionnaire. In this way, the survey data are collected and can be analyzed. 


\section{Results}

As a result of the survey conducted in 2021, some of the obtained results are presented in this part [1]. They are related to the third section of the questionnaire. It is dedicated to the problems of digitized administrative services. The study was conducted using a specialized web application. For this reason, researchers do not need to install any additional and specific software products on their computers, because they can access relevant analytics online [2].

Of the 45 municipalities surveyed in the Republic of Bulgaria, 70.2\% indicated that they had developed and implemented less than 5 new digital services (Fig. 3). Such an answer was indicated by most respondents. Between 6 and 10 services were developed by $21.3 \%$ of the surveyed municipalities. These are mainly the larger municipalities, which are also regional centers. The data obtained on this issue from the questionnaire show that the municipalities are in the process of accelerated digitalization and development of new services.

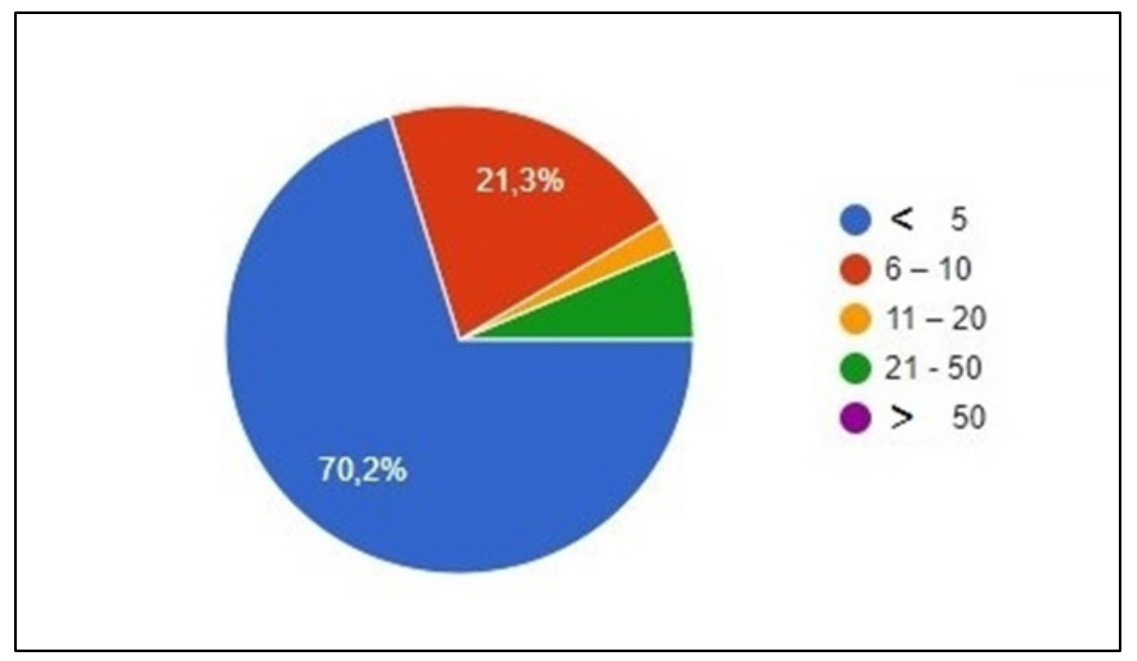

Fig. 3. How many new digital services do you develop and implement in the municipality (average per year)?

The development of new digital services by the local administration depends to a large extent on their use. In these municipalities, where the local population and business have an interest in using new digital channels, preconditions are created for the creation of new administrative services. It is noteworthy that where there is a lower usability of digital services, and their creation is very limited. To study this connection, a similar question is positioned in the questionnaire. The graphical representation of the obtained results is given at Fig. 4. In $91 \%$ of the surveyed municipalities the number of users who use digital administrative services is below 5000. The percentage of municipalities where the number of users is between 5000 and 10000 is very small. In the sample there are several municipalities that are regional centers. And with them this number of users is not very high. 


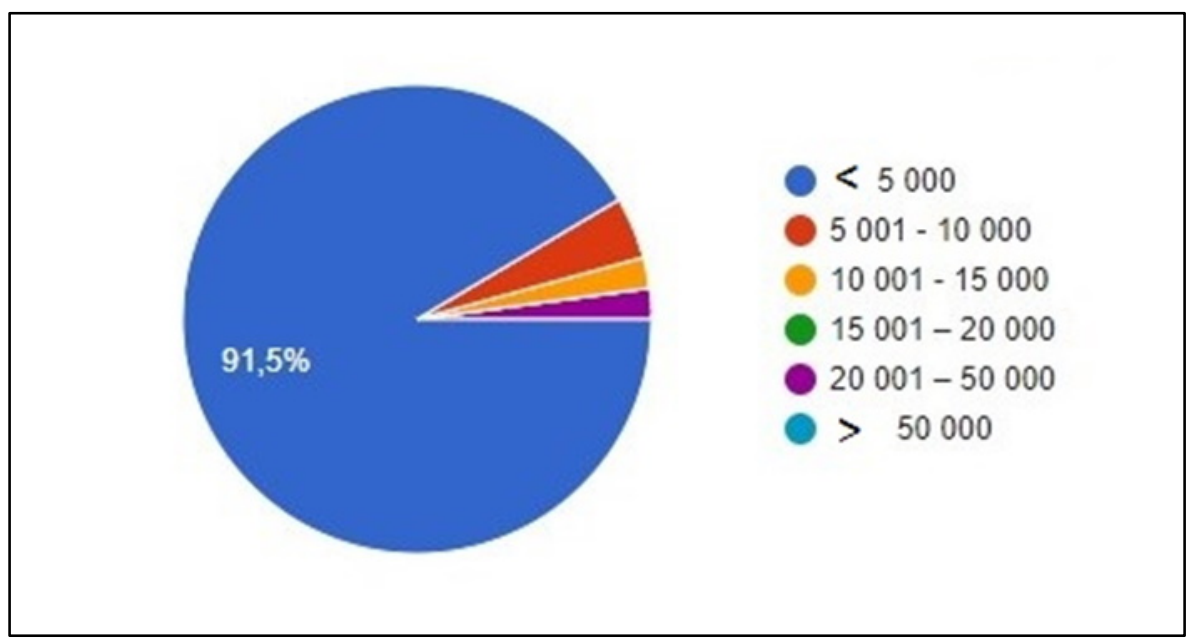

Fig. 4. How many users in the municipality use digital administrative services (average per year)?

Detailing the survey requires looking for an answer to the question of what percentage of administrative services are digitized. The surveyed municipalities have given quite a variety of answers to this question. The answers are wide-ranging. In 33 of all surveyed municipalities the answers given are between $1 \%$ and $50 \%$. This shows that significant efforts are being made to expand the percentage of digitized services. The data obtained on this third question from the questionnaire are given at Fig. 5.

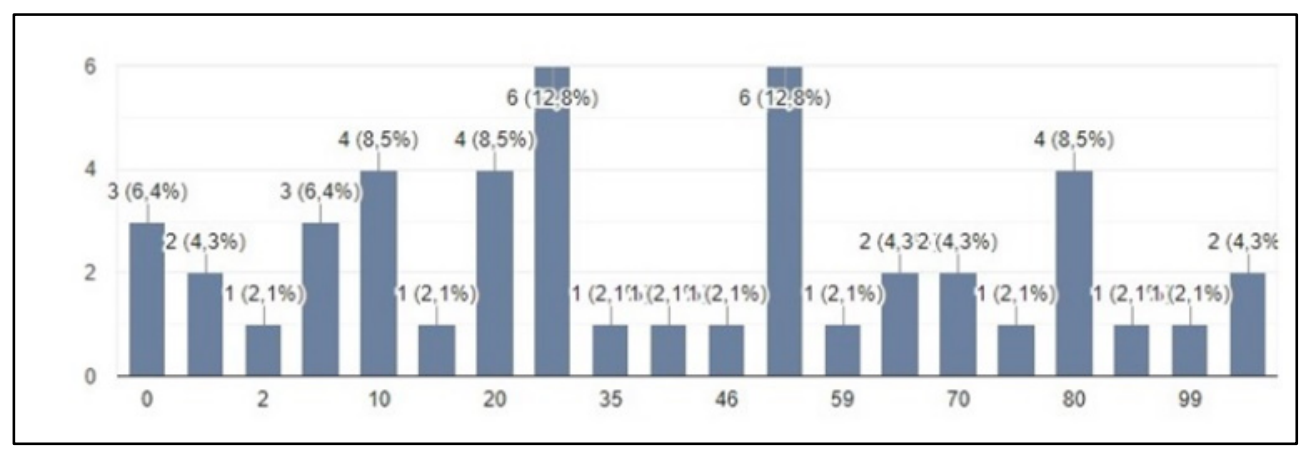

Fig. 5. What percentage of administrative services are digitized?

Along with the digitalization processes, most of the municipalities offer services that have two-way paper communication. With it, users submit a paper application for an administrative service, after which it is necessary to visit the administration and receive a result document. These services require constant visits to the administration offices. This is not convenient for consumers because it takes time and money. For these reasons, such an analysis is interesting for the present study. The data from it are given in fig. 6 . The graph makes a strong impression that in 11 municipalities $(23.4 \%)$ such services are no longer offered. At the same time, in 9 municipalities (19.1\%) these services are about $50 \%$ of all offered administrative services. For the most part, these are again smaller municipalities. 


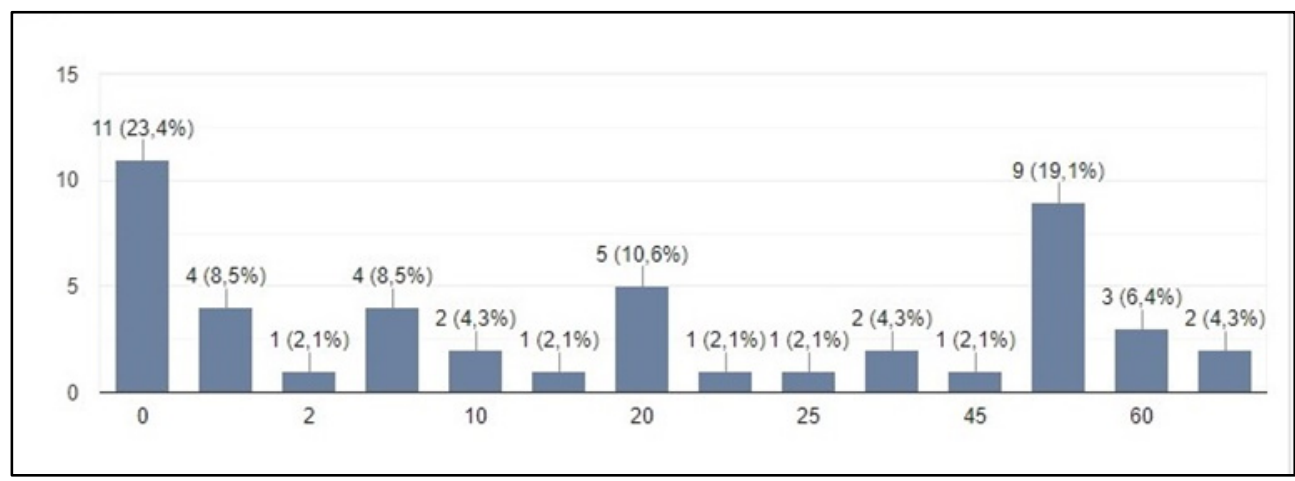

Fig. 6. What percentage of the offered digital administrative services are at the level of two-way communication without the possibility for electronic delivery and electronic payment?

These analyzes show that smaller municipalities need funding and special attention to digitalization processes. This should be done through appropriate programs and projects, as well as through own resources. Much of the solution to this problem is related to investments in human capital and expanding the opportunities for conducting specialized practical training for the administration [3].

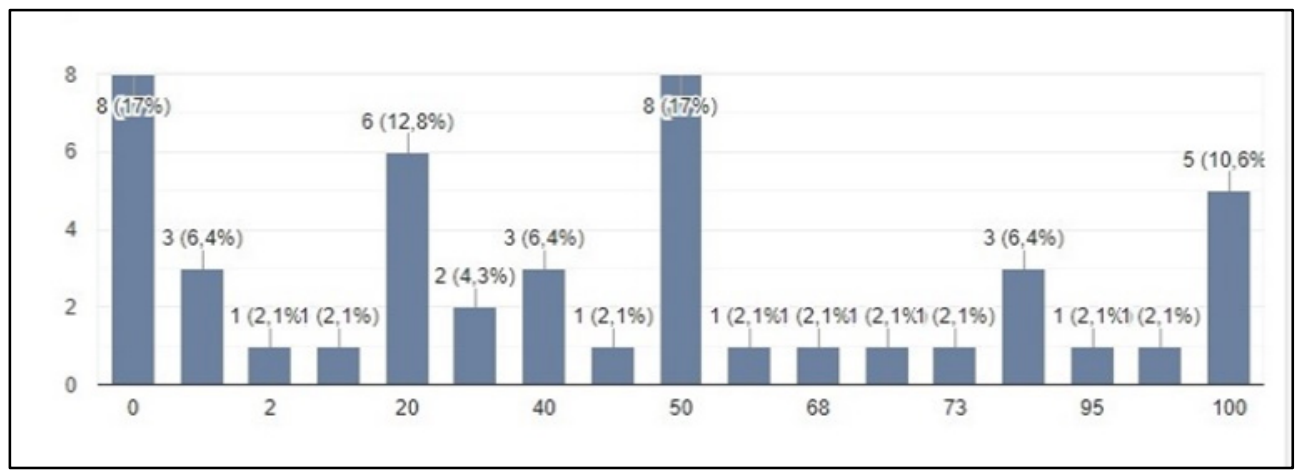

Fig. 7. What percentage of the offered digital administrative services are at the level of two-way communication with the possibility of electronic delivery and electronic payment?

The growth rate of digital services is very important for the creation and implementation of various digitalization policies in local government. It is very difficult to determine without considering the quality and manner of providing the service. For these reasons, the survey provides a special place to study the percentage of "two-tier communication" services that allow electronic payment. Fig. 7 presents a graphical distribution of the results. It shows that in $17 \%$ of the municipalities ( 8 municipalities) such an opportunity is not offered at all. However, with the same number of municipalities, about $50 \%$ of the services offered allow electronic payment. In 5 of the municipalities $100 \%$ of the services can be paid electronically. All this shows the strong pace at which digital payments are entering the local administration. These trends can be expected to deepen in the future.

\section{Conclusions}

The topic of digital administrative services is comprehensive and quite complex. The present study presents the results of a survey conducted among 45 municipalities. The main conclusions from the obtained results are: 
- Significant efforts have been made in recent years to digitize local administrative services;

- In some of the municipalities there are very serious results in terms of number and quality of provided digital administrative services;

- Most of the municipalities have switched or are switching to modern channels for communication with citizens and businesses;

- The studied processes require the allocation of significant financial resources and human capital. This is a difficult task for smaller and remote municipalities;

- A comprehensive policy (financial, administrative, communication) is needed to expand the use of digital channels for communication with local authorities.

This work was supported by the UNWE Research Programme (Research Grant No. NID NI-7/2020).

\section{References}

1. Questionnaire (2021),

https://docs.google.com/forms/d/e/1FAIpQLScatvm2o6h_ZrMnBsOWV49H2Admdaf R0wd0EOqmNfHp-UJEHg/viewform?usp=sf_link, [Accessed: 21 June 2021]

2. P. Milev, Technological approaches for presentation of data analysis in web applications, in Proceedings of the International Conference on Application of Information and Communication Technology and Statistics in Economy and Education, ICAICTSEE, 18-20 October 2018, Sofia, Bulgaria (2020)

3. R. Kirilov, Information Problems of Practical Training, Research Papers of UNWE, 2, 185-196 (2019) 